\title{
Applying the General Regression Neural Network to Ground Motion Prediction Equations of Induced Events in the Legnica-Głogów Copper District in Poland
}

\author{
Jan WISZNIOWSKI \\ Institute of Geophysics, Polish Academy of Sciences, Warsaw, Poland \\ e-mail: jwisz@igf.edu.p
}

\begin{abstract}
This paper presents a study of the nonlinear estimation of the ground motion prediction equation (GMPE) using neural networks. The general regression neural network (GRNN) was chosen for its high learning rate. A separate GRNN was tested as well as a GRNN in cascade connection with linear regression (LR). Measurements of induced seismicity in the Legnica-Głogów Copper District were used in this study. Various sets of input variables were tested. The basic variables used in every case were seismic energy and epicentral distance, while the additional variables were the location of the epicenter, the location of the seismic station, and the direction towards the epicenter. The GRNN improves the GMPE. The best results were obtained when the epicenter location was used as an additional input. The GRNN model was analysed for how it can improve the GMPE with respect to LR. The bootstrap resampling method was used for this purpose. It proved the statistical significance of the improvement of the GMPE. Additionally, this method allows the determination of smoothness parameters for the GRNN. Pa-
\end{abstract}

Ownership: Institute of Geophysics, Polish Academy of Sciences

(C) 2016 Wiszniowski. This is an open access article distributed under the Creative Commons Attribution-NonCommercial-NoDerivs license (http://creativecommons.org/licenses/by-nc$n d / 3.0 /)$. 
rameters derived through this method have better generalisation capabilities than the smoothness parameters estimated using the holdout method.

Key words: ground motion prediction equation, artificial neural network, general regression neural network.

\section{INTRODUCTION}

Since the beginning of seismic hazard analysis, ground motion prediction equations (GMPEs) have been effectively used to estimate ground motions in deterministic and probabilistic seismic hazard analyses. The basic method of estimation of GMPE is linear regression (LR), where magnitude and the logarithm of distance are independent variables, and the logarithm of peak ground acceleration is the dependent variable (Cornell 1968). Instead of magnitude, the logarithm of energy can also be used (e.g., Golik and Mendecki 2012, Lasocki 2013).

While there are GMPEs with more expanded formulae (e.g., Douglas 2011), which incorporate higher powers of magnitude (e.g., Trifunac and Brady 1976, Joyner and Boore 1988, Akkar and Bommer 2010) and complex functions incorporating source models (e.g., Abrahamson and Silva 2008, Boore and Atkinson 2008, Campbell and Bozorgnia 2008), the use of artificial neural networks (ANNs) to estimate the GMPE does not require knowledge of these models.

Basically, GMPEs vary considerably depending on the seismic zone (Douglas 2011), and it is common to perform GMPE estimation for each particular case individually. For induced seismicity, the most effective GMPEs are often basic LR models (Golik and Mendecki 2012, Lasocki 2013).

ANNs have been chosen as a tool to answer the question of whether using nonlinear regression can help create a model that results in a significantly better description of the predicted mean of the GMPE.

For nonlinear estimation, three types of neural networks are used most frequently: multilayer perceptrons (MLPs) with one or two hidden layers (Pozos-Estrada et al. 2014), networks with radial based functions (RBFs), and general regression neural networks (GRNNs). All three solutions have been applied to the GMPE (Günaydin and Günaydin 2008), with MLP being used most often (Güllü and Erçcelebi 2007, Derras et al. 2012, Hong et al. 2012, Arjun 2013). The magnitude and epicentral or hypocentral distance have been used as input data for the GMPE estimation with the help of ANNs. The additional input data that have also been used are: focal depth (Pozos-Estrada et al. 2014); shear wave velocity down to $30 \mathrm{~m}\left(\mathrm{~V}_{\mathrm{S} 30}\right)$ and focal depth (Hong et al. 2012, Derras et al. 2012); the thickness of the sedimentary layers below the site down to a shear wave velocity equal to 
$800 \mathrm{~m} / \mathrm{s}$, and the corresponding resonant frequency (Derras and Bekkouche 2011); the fundamental resonance frequency, as determined by the horizontal-to-vertical (H/V) spectral ratio technique (Derras et al. 2012); site conditions (Güllü and Erçelebi 2007, Günaydin and Günaydin 2008); and the average values of shear wave velocity, primary wave velocity, standard penetration test blow count, and the density of soil (Arjun 2013).

For this paper we chose the GRNN method, because it is fast-learning and trends toward the optimal regressional surface. In the case of testing the usefulness of ANN for GMPE estimation, using the GRNN gives a quicker result, without the need to analyse whether the ANN is optimally trained.

Recently, GRNN networks have been applied for solving different problems in the fields of seismic hazard and earthquake engineering. García et al. (2003) and Derras and Bekkouche (2011) applied GRNN together with other ANN methods for estimation of the GMPE. Yaghmaei-Sabegh and Tsang $(2011,2014)$ applied the GRNN as well as probabilistic neural network (PNN) for site classification based on an $\mathrm{H} / \mathrm{V}$ spectral ratio technique. Yaghmaei-Sabegh (2012) also employed the GRNN for ranking and weighting the GMPE models in the logic tree. Hanna et al. (2007) employed the GRNN for assessing liquefaction in soil deposits.

Although earlier studies have not shown any better effectiveness of the GMPE estimated by GRNN than the MLP (García et al. 2003, Derras and Bekkouche 2011), the possibility of quick learning and the uniqueness of training allow the examination of the distribution of GMPE model error using the bootstrap method, and testing the statistical significance of the improvement of the GMPE. The effectiveness of the GMPE estimated by GRNN is improved by applying the GRNN in connection with the LR, where the GRNN reduces the error of LR.

Data on induced seismicity in the Legnica-Głogów Copper District (LGCD) in Poland were used in this study. Tremors with energy of up to $10^{9} \mathrm{~J}$ occur in this region, and generate motions of up to $3 \mathrm{~m} / \mathrm{s}^{2}$. The GMPEs for this region have been previously estimated based on LR methods (Lasocki 2013).

\section{THE GRNN}

The concept of the GRNN (Specht 1991) is based on computing the conditional mean of $y$, given $X$, according to the formula

$$
E(y \mid X)=\frac{\int_{-\infty}^{+\infty} y f(X \mid y) d y}{\int_{-\infty}^{+\infty} f(X \mid y) d y},
$$


where $X$ is a particular measured value of the random variable $x$ and $f(X \mid y)$ is the condition probability density function of $X$ given $y$. Because $f(X \mid y)$ is not known, usually the estimator proposed by Parzen (1962) is used.

In the implementation of the GRNN, the integration is performed by summation, according to the formula:

$$
\hat{Y}(X)=\frac{\sum_{i=1}^{n} Y_{i} p\left(X \mid Y_{i}\right)}{\sum_{i=1}^{n} p\left(X \mid Y_{i}\right)},
$$

where $n$ is the number of samples used for learning.

The GRNN consists of four layers (Fig. 1): input, pattern, summation and output. The input layer is made up of units corresponding to each of the independent variables used to estimate the ground motion. The number of units in the pattern layer is the same as the number of samples in the training set. The $i^{\text {th }}$ unit in the pattern layer, which corresponds to the $i^{\text {th }}$ sample in the training set, computes $p\left(\boldsymbol{x} \mid y_{i}\right)$ according to the formula:

$$
p\left(x \mid y_{i}\right)=\exp \left(\frac{D_{i}^{2}}{\sigma^{2}}\right),
$$

where $\sigma$ is the smoothness parameter of the GRNN and $D_{i}$ is the distance between the $i^{\text {th }}$ sample and the value in the input layer. The summation layer consists of two units that compute the numerator

$$
S=\sum_{i=1}^{n} y_{i} p\left(x \mid y_{i}\right)
$$

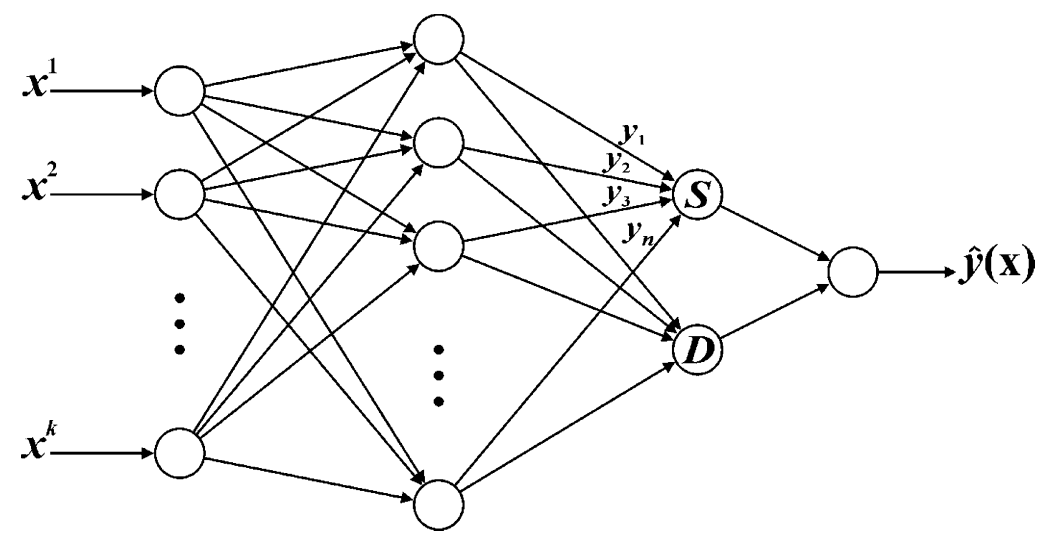

Fig. 1. Diagram of the GRNN. 
and the denominator:

$$
D=\sum_{i=1}^{n} p\left(x \mid y_{i}\right) .
$$

The output layer realises the division:

$$
\hat{y}(x)=\frac{S}{D} .
$$

The smoothness parameter $\sigma$ is the only value selected during the network learning process. The holdout method proposed by Specht (1991) consists of removing one sample at a time and constructing a network based on all of the other samples. Then, the network is used to calculate the squared error between the expected $Y$ and the estimated $\hat{Y}$ for the removed sample. By repeating this process for each sample, and for the particular value of $\sigma$, the sum of squared errors (SSE) for all samples is calculated. The value of $\sigma$ with the smallest SSE is used in the GRNN. In the following sections, the other method for determining $\sigma$ is proposed. It is based on the bootstrap method.

As a preprocessing step, we scale all input variables such that they have approximately the same ranges or variances. The need for this process stems from the fact that the probability density function $f(X \mid y)$ is to be estimated using (3) with only one smoothness parameter (Specht 1991). Therefore, the metric in the form

$$
D_{i}^{2}=\sum_{j=1}^{k} \frac{\left(x^{j}-x_{i}^{j}\right)^{2}}{\operatorname{Var}\left(X^{j}\right)}
$$

was used, where $k$ is the number of input variables (Fig. 1).

The GRNN has advantages and disadvantages when compared to MLPs. The GRNN has a solid mathematical background (Wasserman 1993). It is a one-pass learning algorithm (i.e., lazy learning). Prediction is unique, which means that it is not dependent on the training procedure or the initial conditions. On the other hand, the GRNN requires more memory space to store the model and is slower than MLPs when classifying new cases.

\section{DATA USED TO TEST THE GRNN}

Copper ore extraction by three underground mines in the LGCD in southwest Poland, which takes place in hard rocks at depths of 800-1100 m, generating earthquakes that can exceed 4.5 of local magnitude and $10^{9} \mathrm{~J}$ of energy, often significantly affects buildings and other surface structures. The 


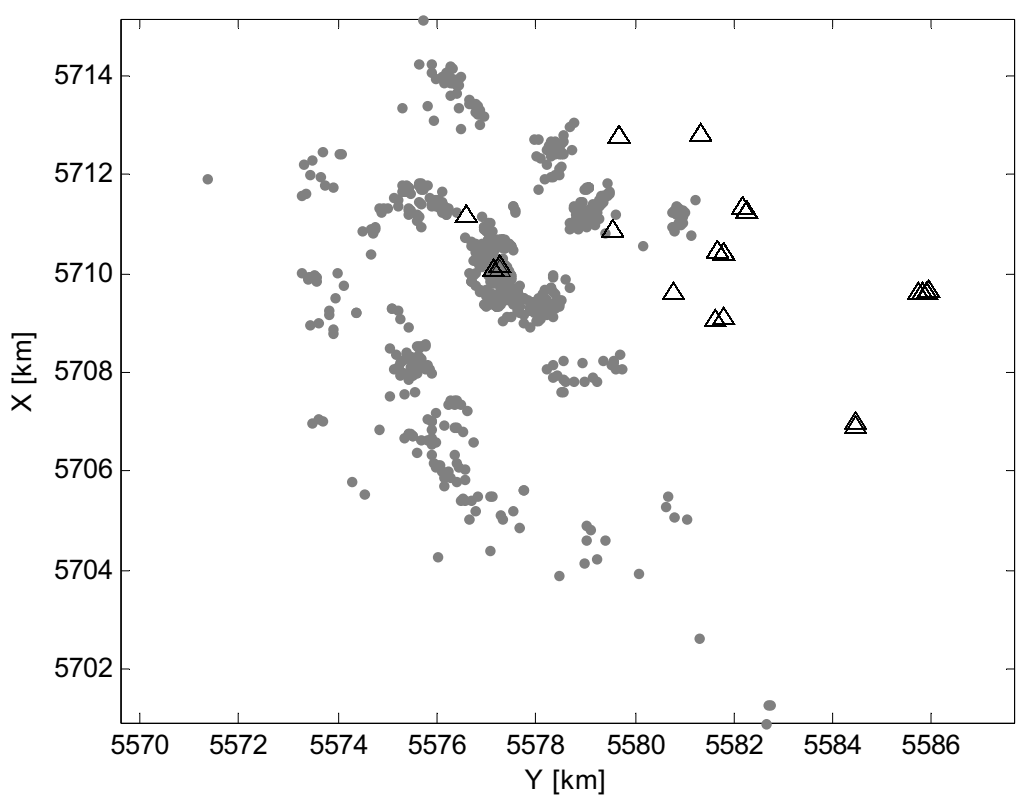

Fig. 2. Distribution of earthquakes recorded in the LGCD, 2001-2012. Dots - earthquakes, triangles - stations.

strongest events are capable of producing a peak ground acceleration (PGA) of more than $3 \mathrm{~m} / \mathrm{s}^{2}$.

A GMPE is a function of values describing an event, site, and source-tosite route. For the LGCD, only data on ground motion, seismic energy, the location of the epicenter, and the location of the station are provided and can be used for estimation of the GMPE. The data consisted of 2991 ground motion measurements of 904 events recorded by up to 11 accelerometers (Fig. 2 ) in the period from April 2001 to November 2012. For the GMPE estimation, we used values that were functions of the original values:

- $\log E$ - the logarithm of energy,

- $\log R$ - the logarithm of distance, where

$$
R=\sqrt{r^{2}+h_{0}^{2}},
$$

and $r$ is the source-receiver epicentral distance calculated from the coordinates of the epicenter and station, whereas the coefficient $h_{0}$ is the common depth factor introduced by Joyner and Boore (1993). The value $h_{0}=800$ was chosen by minimising the error of linear regression of $\log E$ and $\log R$. The additional input variables are: 

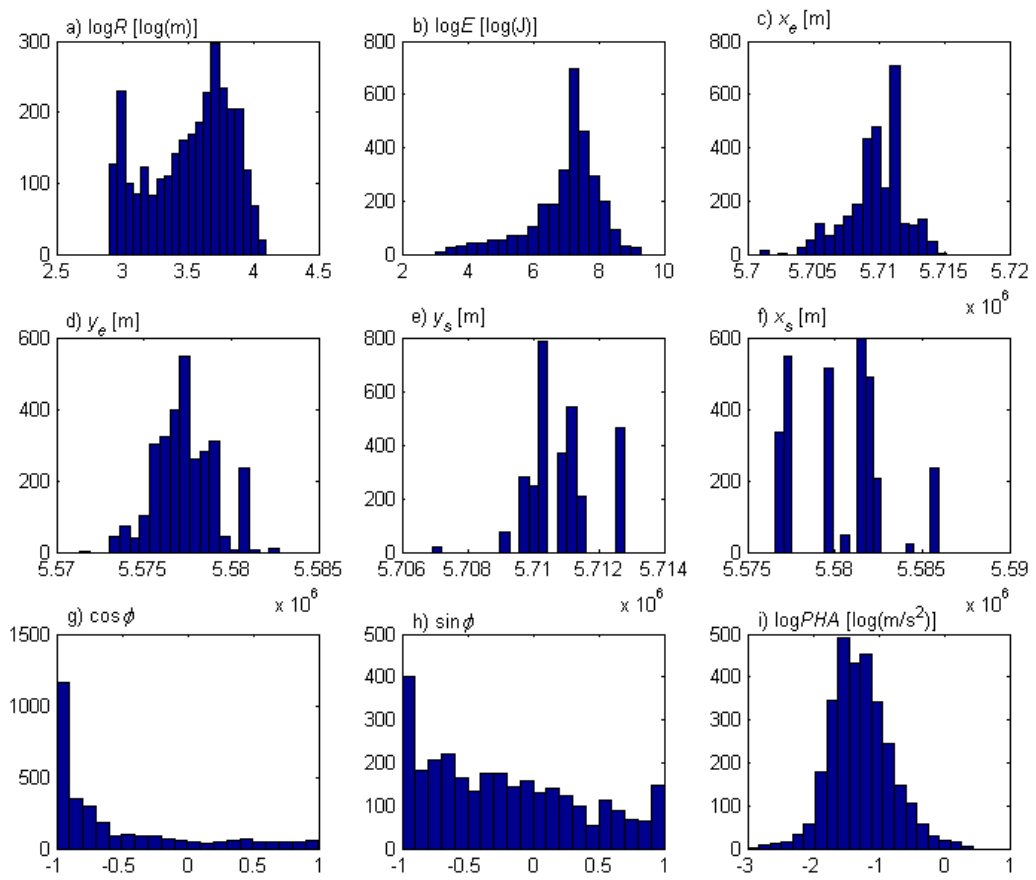

Fig. 3. Histograms of inputs (a-h) and output of the GRNN (i): (a) logarithms of distances; (b) logarithms of energies; (c \& d) coordinates of the epicenters; (e \& f) coordinates of the seismic stations; ( $g$ ) cosines of radial directions; (h) sines of radial directions; (i) logarithms of peak horizontal accelerations.

- $x_{e}, y_{e}-$ the coordinates of the epicenters,

- $x_{s}, y_{s}-$ the coordinates of the seismic stations,

- $\cos \varphi, \sin \varphi-$ the cosines and sines of radial direction - instead of $\cos \varphi$ and $\sin \varphi$, angles $\varphi$ could be used as well; however, the cosine and sine functions vary smoothly when the angle changes from $360^{\circ}$ to $0^{\circ}$.

The output value was the logarithm of peak horizontal component of ground acceleration (i.e., Peak Horizontal Acceleration, $P H A$ ). Figure 3 shows individual histograms of these values, while Fig. 2 better illustrates the distribution of coordinates $\left(x_{e}, y_{e}\right.$ and $\left.x_{s}, y_{s}\right)$ as they are, in fact, 2D variables.

The dataset is divided randomly into training and test sets during the bootstrap test. Any outliers are not removed.

\section{APPLYING THE GRNN TO THE GMPE}

The GRNN was tested both separately and in cascade with an LR estimator. Several GMPE models were compared (Fig. 4). 
a)

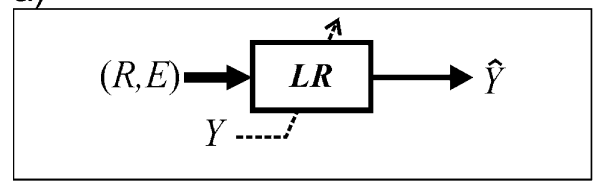

c)

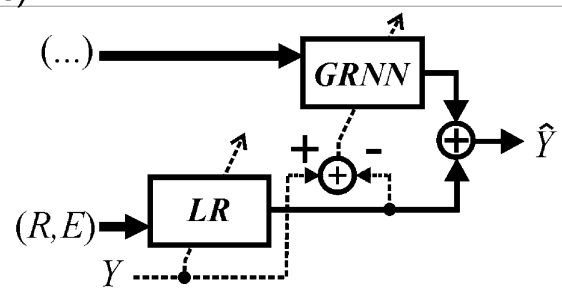

b)

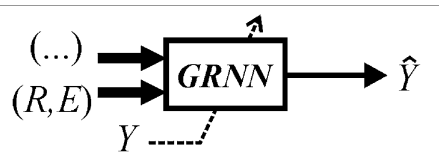

d)

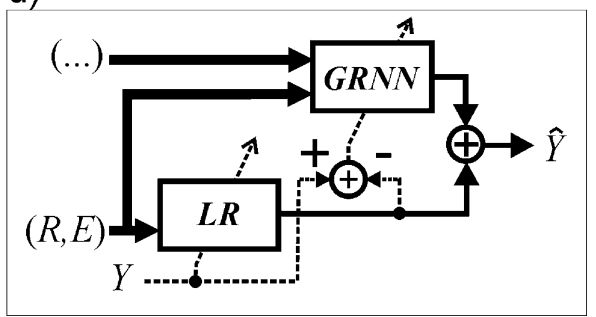

Fig. 4. Diagrams of the GMPE models: (a) LR of $\log R$ and $\log E$; (b) separate GRNN, where $\log R$ and $\log E$ are some of the inputs; (c) GRNN in cascade with LR, where $\log R$ and $\log E$ are not inputs of the GRNN; (d) GRNN in cascade with LR, where $\log R$ and $\log E$ are inputs of the GRNN.

The reference model is the LR model (Fig. 4a), which is commonly used for GMPE (Douglas 2011), especially in the case of mining induced seismicity (Golik and Mendecki 2012, Lasocki 2013). The effectiveness of all GRNN models is compared to that of the LR. The considered form of the LR model is:

$$
\log a=\alpha+\beta \log E+\gamma \log R,
$$

where $a$ is the PHA in $\mathrm{m} / \mathrm{s}^{2}$ estimated from the model. The form of LR was chosen because it was applied to the GMPE in the LGCD by Lasocki (2013). The estimated parameters of LR were:

$$
\begin{gathered}
\alpha=0.41, \\
\beta=0.47, \\
\gamma=-1.42, \\
R^{2}=0.731 .
\end{gathered}
$$

The value of the $R^{2}$ is typical of mining induced GMPEs. The coefficient of determination obtained by Lasocki (2013), for the LR model covering different parts of the LGCD and estimated from a smaller amount of data (1818 records), was lower $\left(R^{2}=0.532\right)$. In the case of induced seismicity in the Upper Silesian Coal Basin in Poland (Golik and Mendecki 2012), the coefficients of determination of GMPE models are in the range of 0.64 (region of "Bielszowice" coal mine) to 0.79 (region of "Ziemowit" coal mine). The er- 
ror of LR (10) model does not have a normal distribution (Fig. 5). It did not pass Lilliefors's normality test $(\mathrm{p}=0.007)$.

The next tested model was the separate GRNN (Fig. 4b). Its inputs were $\log R, \log E$, and combinations of the following pairs of variables (described as '(...)' in Fig. 4):

$$
\left(x_{e}, y_{e}\right),\left(x_{s}, y_{s}\right),(\cos \varphi, \sin \varphi) .
$$

However, $\log R, \cos \varphi$ and $\sin \varphi$ are functions of $x_{e}, y_{e}$ and $x_{s}, y_{s}$. Therefore, another GMPE model, in the form of the GRNN $\left(\log E, x_{e}, y_{e}, x_{s}, y_{s}\right)$, was also analysed. It was the only model in which the distance was not given as $\log R$.

The models in Figs. $4 \mathrm{c}$ and $4 \mathrm{~d}$ are combinations of LR and the GRNN. Both models consist of two components in cascade. The first component is the LR. Its inputs are only $\log R$ and $\log E$. Its output is the linear prediction of ground motion. The second component is the GRNN. It is trained by residuals of the LR. The output of the whole model is the sum of the outputs of the LR and GRNN. The GMPE model, in which the GRNN improves results of the LR, is referred to as $G_{R N N}$. In the model presented in Fig. 4c, the GRNN input incorporates only combinations of inputs (11), whereas in the

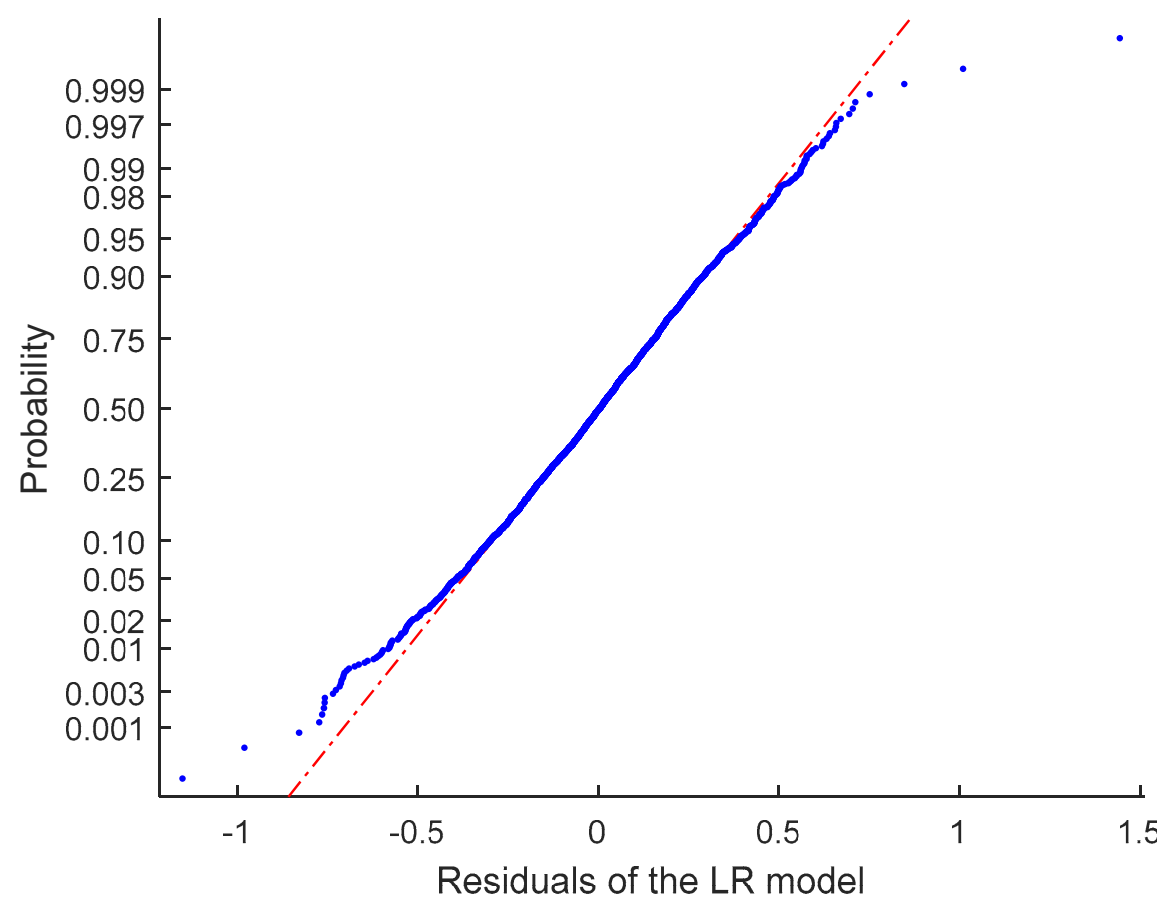

Fig. 5. Normal probability plot for the LR model. 
model presented in Fig. 4d, the inputs $\log R$ and $\log E$ are also included in GRNN. As the smoothness parameter increases, the output of the GRNN approaches the mean value of $\log P H A$, whereas $G_{R N N}$ approaches the result of the LR. Therefore, the GRNN $\mathrm{R}_{\mathrm{R}}$ is less sensitive to large values of $\sigma$.

Table 1

Results of holdout method Optimum smoothness parameter values obtained with the help of the holdout method and corresponding residual sum of squares, and the coefficient of determination for various GMPE models utilising the GRNN and various combinations of inputs

\begin{tabular}{|c|c|c|c|c|}
\hline \multicolumn{2}{|r|}{ GMPE model } & $\sigma$ & $S S_{\text {res }}$ & $R^{2}$ \\
\hline \multirow{8}{*}{$\begin{array}{c}\text { Separate } \\
\text { GRNN } \\
\text { (Fig. 4b) }\end{array}$} & GRNN $(\log E, \log R)$ & 0.096 & 142.84 & 0.77 \\
\hline & $\begin{array}{l}\operatorname{GRNN}\left(\log E, \log R, x_{e}, y_{e}, x_{s}, y_{s}, \cos \varphi,\right. \\
\sin \varphi)\end{array}$ & 0.235 & 115.23 & 0.81 \\
\hline & $\operatorname{GRNN}\left(\log E, \log R, x_{e}, y_{e}, x_{s}, y_{s}\right)$ & 0.175 & 110.12 & 0.82 \\
\hline & $\operatorname{GRNN}\left(\log E, \log R, x_{e}, y_{e}, \cos \varphi, \sin \varphi\right)$ & 0.224 & 113.57 & 0.82 \\
\hline & $\operatorname{GRNN}\left(\log E, \log R, x_{s}, y_{s}, \cos \varphi, \sin \varphi\right)$ & 0.222 & 118.76 & 0.81 \\
\hline & $\operatorname{GRNN}\left(\log E, \log R, x_{e}, y_{e}\right)$ & 0.138 & 104.14 & 0.83 \\
\hline & $\mathrm{GRNN}\left(\log E, \log R, x_{s}, y_{s}\right)$ & 0.113 & 119.93 & 0.81 \\
\hline & GRNN $(\log E, \log R, \cos \varphi, \sin \varphi)$ & 0.166 & 129.77 & 0.79 \\
\hline \multirow{9}{*}{$\begin{array}{c}\mathrm{GRNN}_{\mathrm{R}} \\
\text { with inputs } \\
\log \mathrm{E} \text { and } \\
\log R \\
\text { (Fig. 4d) }\end{array}$} & $\mathrm{GRNN}\left(\log E, x_{e}, y_{e}, x_{s}, y_{s}\right)$ & 0.147 & 109.87 & 0.82 \\
\hline & $\mathrm{GRNN}_{\mathrm{R}}(\log E, \log R)$ & 0.100 & 142.19 & 0.77 \\
\hline & $\begin{array}{l}\operatorname{GRNN}_{\mathrm{R}}\left(\log E, \log R, x_{e}, y_{e}, x_{s}, y_{s}, \cos \varphi,\right. \\
\sin \varphi)\end{array}$ & 0.294 & 106.22 & 0.83 \\
\hline & $\operatorname{GRNN}_{\mathrm{R}}\left(\log E, \log R, x_{e}, y_{e}, x_{s}, y_{s}\right)$ & 0.180 & 104.21 & 0.83 \\
\hline & $\operatorname{GRNN}_{\mathrm{R}}\left(\log E, \log R, x_{e}, y_{e}, \cos \varphi, \sin \varphi\right)$ & 0.271 & 105.32 & 0.83 \\
\hline & $\mathrm{GRNN}_{\mathrm{R}}\left(\log E, \log R, x_{s}, y_{s}, \cos \varphi, \sin \varphi\right)$ & 0.264 & 111.41 & 0.82 \\
\hline & $\mathrm{GRNN}_{\mathrm{R}}\left(\log E, \log R, x_{e}, y_{e}\right)$ & 0.141 & 100.93 & 0.84 \\
\hline & $\operatorname{GRNN}_{\mathrm{R}}\left(\log E, \log R, x_{s}, y_{s}\right)$ & 0.114 & 120.16 & 0.81 \\
\hline & $\operatorname{GRNN}_{\mathrm{R}}(\log E, \log R \cos \varphi, \sin \varphi)$ & 0.183 & 125.05 & 0.80 \\
\hline \multirow{7}{*}{$\begin{array}{l}\mathrm{GRNN}_{\mathrm{R}} \\
\text { without in- } \\
\text { puts } \log E \text {, } \\
\text { and } \log R \\
\text { (Fig. 4c) }\end{array}$} & $\mathrm{GRNN}_{\mathrm{R}}\left(x_{e}, y_{e}, x_{s}, y_{s}, \cos \varphi, \sin \varphi\right)$ & 0.102 & 115.48 & 0.82 \\
\hline & $\operatorname{GRNN}_{\mathrm{R}}\left(x_{e}, y_{e}, x_{s}, y_{s}\right)$ & 0.069 & 111.24 & 0.82 \\
\hline & $\operatorname{GRNN}_{\mathrm{R}}\left(x_{e}, y_{e}, \cos \varphi, \sin \varphi\right)$ & 0.097 & 115.80 & 0.82 \\
\hline & $\mathrm{GRNN}_{\mathrm{R}}\left(x_{s}, y_{s}, \cos \varphi, \sin \varphi\right)$ & 0.088 & 147.43 & 0.76 \\
\hline & $\operatorname{GRNN}_{\mathrm{R}}\left(x_{e}, y_{e}\right)$ & 0.012 & 112.13 & 0.82 \\
\hline & $\operatorname{GRNN}_{\mathrm{R}}\left(x_{s}, y_{s}\right)$ & 0.039 & 161.03 & 0.74 \\
\hline & $\operatorname{GRNN}_{\mathrm{R}}(\cos \varphi, \sin \varphi)$ & 0.107 & 163.99 & 0.74 \\
\hline
\end{tabular}


The quality of methods is assessed based on the residual sum of squares, $S S_{\text {res }}$, and the coefficient of determination, $R^{2} . S S_{\text {res }}$ is defined as

$$
S S_{\text {res }}=\sum_{i=1}^{n}\left(\hat{y}_{i}-y_{i}\right)^{2}
$$

where $y_{i}$ is the measured output and $\hat{y}_{i}$ is the output of the GMPE model. $R^{2}$ is defined as

$$
R^{2}=1-\frac{S S_{r e s}}{S S_{t o t}},
$$

where $S S_{t o t}$ is the total sum of squares defined as:

$$
S S_{t o t}=\sum_{i=1}^{n}\left(y_{i}-\bar{y}\right)^{2},
$$

and:

$$
\bar{y}=\frac{1}{n} \sum_{i=1}^{n} y_{i}
$$

Table 1 shows the $S S_{\text {res }}$ and $\mathrm{R}^{2}$ for the smoothness parameter that was selected using the holdout method (Specht 1991) for the analysed models and for the analysed GMPE inputs. The best prediction improvement for all the GRNN models was obtained when the epicenter of the earthquake was incorporated as an input value. Prediction was also improved when the only GRNN inputs were $\log R$ and $\log E$. The model with no $\log R$ as input $\left(\mathrm{GRNN}\left(\log E, x_{\mathrm{e}}, y_{\mathrm{e}}, x_{s}, y_{s}\right)\right)$ also yielded good results. Using too many input parameters did not improve the GMPE. In the case of epicenter location $\left(x_{\mathrm{e}}\right.$, $\left.y_{\mathrm{e}}\right)$, the quality of the results was reduced when the station location $\left(x_{s}, y_{s}\right)$, or the angular coefficients $(\cos \varphi, \sin \varphi)$, were included. However, the best results were obtained for $\mathrm{GRNN}_{\mathrm{R}}$ with inputs $\log E$ and $\log R$, which suggests that adding the inputs $\log R$ and $\log E$ had no negative impact on prediction.

\section{TESTING THE SIGNIFICANCE OF GMPE IMPROVEMENT FOR THE GRNN}

Due to the differences in the LR and GRNN methodologies, a method based on the bootstrap (Efron 1979) was chosen to compare them. The full list of records was randomly divided into the subset for training the network and the test subset. The training subset contained one-quarter of all records. The remaining validation subset was used for estimation of the prediction error (Moody 1994). This operation was performed 1000 times for each GRNN model and for each set of inputs. The results are presented in Fig. 6. The 
bootstrap tests were performed for various values of $\sigma$ that gave the distribution of $R^{2}$ as a function of $\sigma$. The mean values of $R^{2}$ of GRNNs are shown as solid lines, whereas the $5^{\text {th }}$ and $95^{\text {th }}$ percentiles of $R^{2}$ are shown as dotted lines.

The LR was estimated and tested in an analogous way, and the following result was obtained:

- The mean value of $R^{2}$ was 0.7281 ;

- The $5^{\text {th }}$ percentile of $R^{2}$ was 0.7202 ;

- The $95^{\text {th }}$ percentile of $R^{2}$ was 0.7334 .

a) GRNN( $\log R, \log E)$

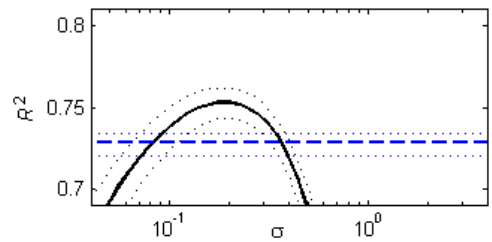

c) GRNN( $\left.\log R, \log E_{1} \cos \phi, \sin \phi\right)$

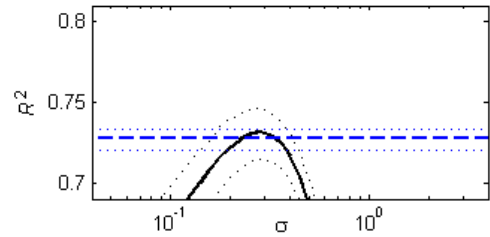

e) GRNN( $\left.\log R, \log E, y_{e^{\prime}} y_{e^{\prime}} y_{s^{\prime}} y_{s}\right)$

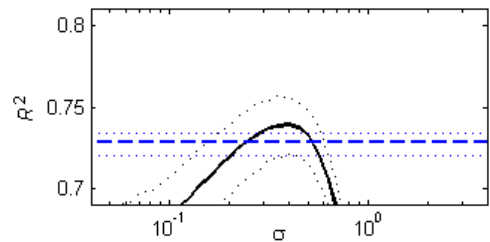

g) GRNN( $\left.\log R, \log E_{1} y_{e}, y_{e}, \cos \phi, \sin \phi\right)$

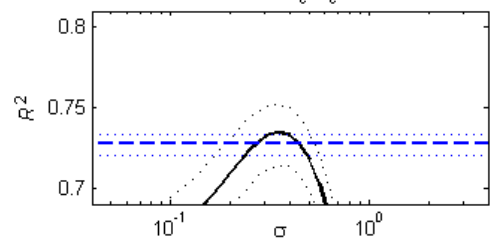

b) GRNN( $\left.\log R, \log E, y_{s^{\prime}} y_{s}\right)$

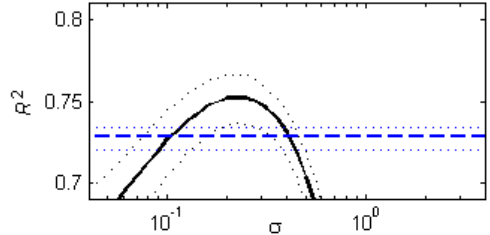

d) GRNN( $\left.\log R, \log E, y_{e}, y_{e}\right)$

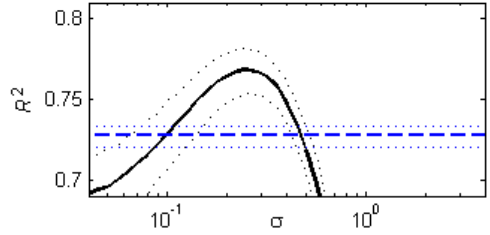

f) GRNN(log $\left.R, \log E, y_{s^{\prime}} y_{s^{\prime}} \cos \phi, \sin \phi\right)$

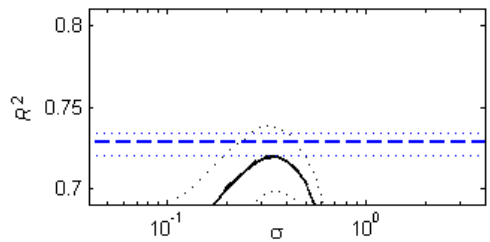

h) GRNN( $\left.\log R, \log E_{1} y_{e}, y_{e^{\prime}} y_{s^{\prime}} y_{s^{\prime}} \cos \phi, \sin \phi\right)$

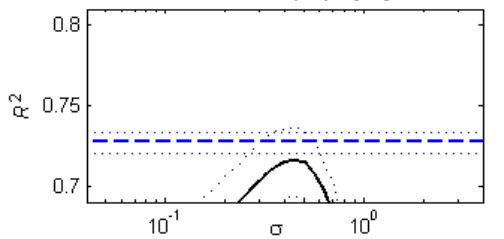

Fig. 6. Values of the coefficient of determination for bootstrap testing of the GRNN as a function of the smoothness parameter. Solid lines - mean $R^{2}$ calculated for the testing set (data not used for training the GRNN); dashed lines - mean $R^{2}$ calculated using LR; dotted lines $-5^{\text {th }}$ and $95^{\text {th }}$ percentiles of $R^{2}$. a-h: results for the separate GRNN (Fig. 4b). Continued on next page. 

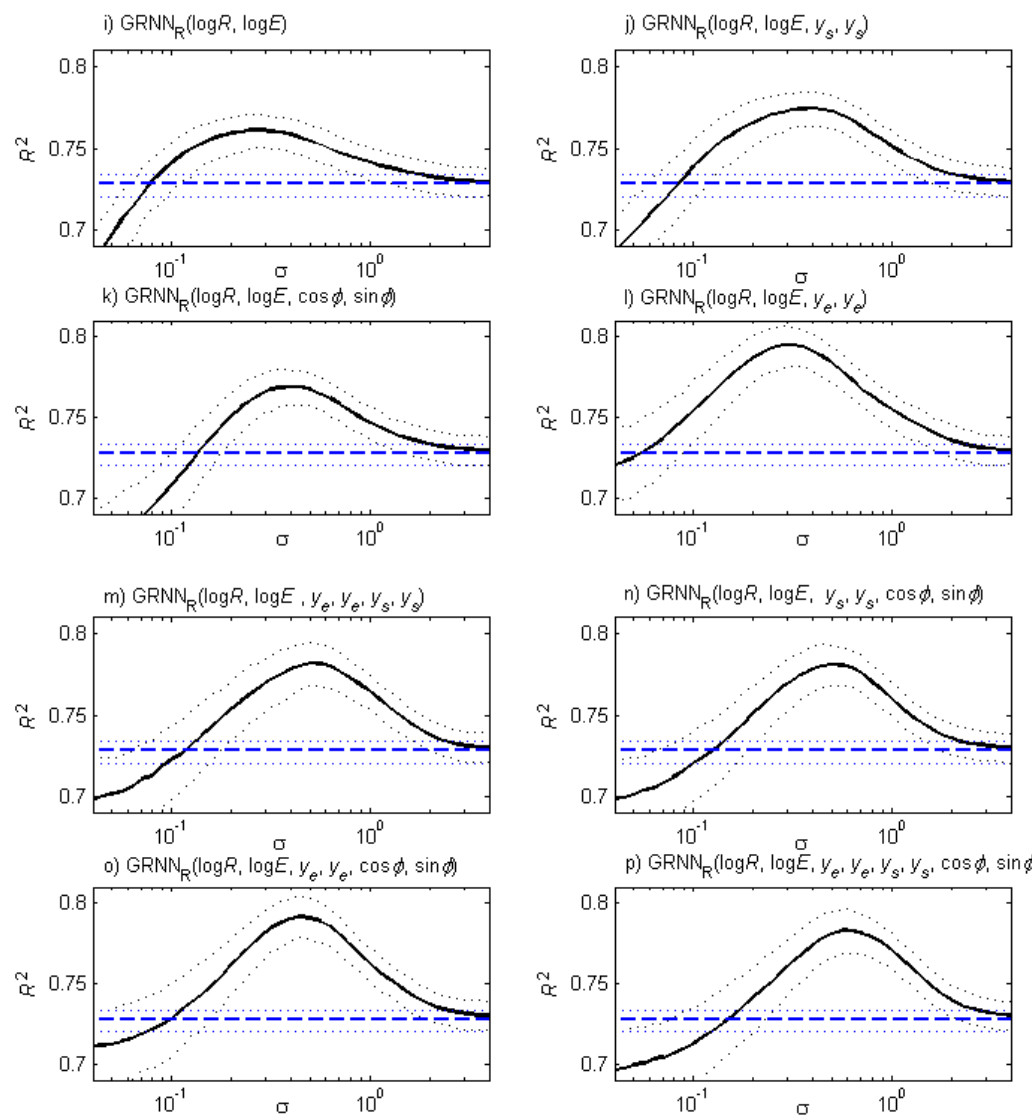

Fig. 6 continuation: Solid lines - mean $R^{2}$ calculated for the testing set (data not used for training the GRNN); dashed lines - mean $R^{2}$ calculated using LR; dotted lines $-5^{\text {th }}$ and $95^{\text {th }}$ percentiles of $R^{2}$. i-p: Results for the cascade GRNN with $\log E$ and $\log R$ as $\mathrm{GRNN}_{\mathrm{R}}$ inputs (Fig. $4 \mathrm{~d}$ ). Continued on next page.

The mean value of $R^{2}$ of the LR is shown as dashed lines in Fig. 6, whereas the $5^{\text {th }}$ and $95^{\text {th }}$ percentiles of $R^{2}$ of the LR are shown as dotted lines.

Marking the $5^{\text {th }}$ percentile for the GRNN and the $95^{\text {th }}$ percentile for the LR in Fig. 6 allows us to conclude whether or not the estimation made using the GRNN is statistically significantly better than that made using LR, and for which values of $\sigma$. If we take an optimum $\sigma$ that maximises the $5^{\text {th }}$ percentile of $R^{2}$, the bootstrap method can be used to assess the minimum GRNN improvement of the GMPE estimation with the probability is greater than $95 \%$, and to select the corresponding value of $\sigma$. 

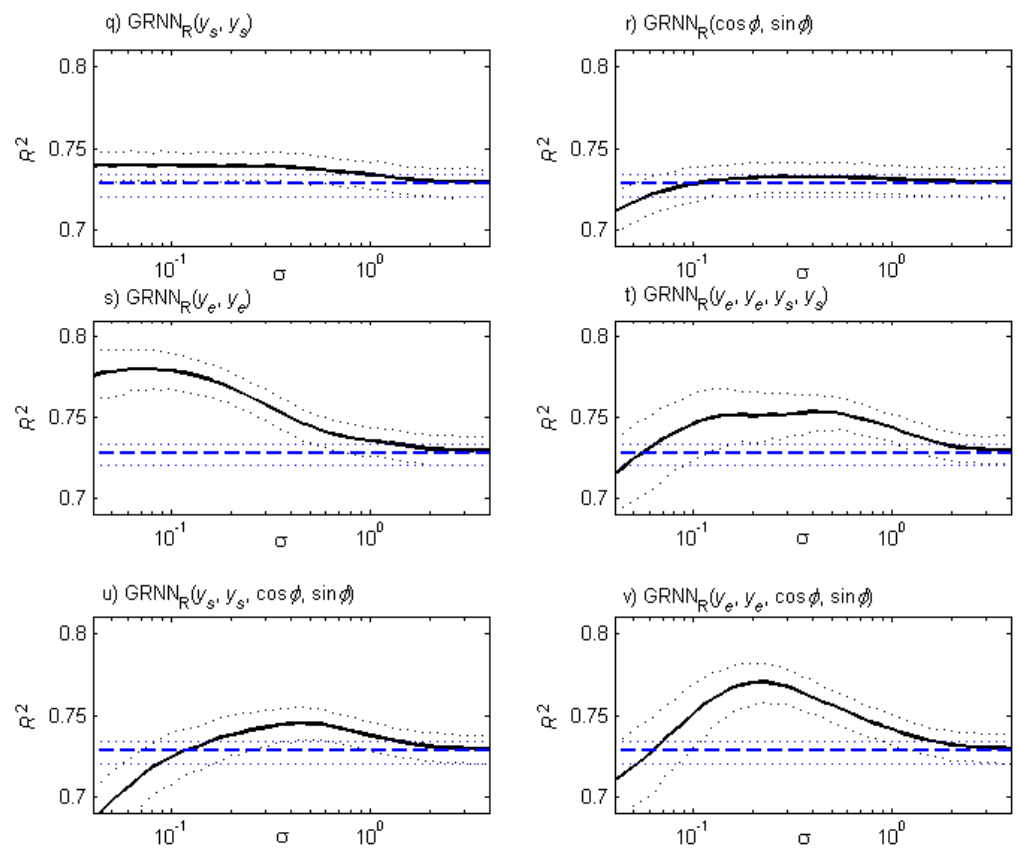

w) $\operatorname{GRNN}_{R}\left(y_{e}, y_{e}, y_{s^{\prime}} y_{s^{\prime}} \cos \phi, \sin \phi\right)$
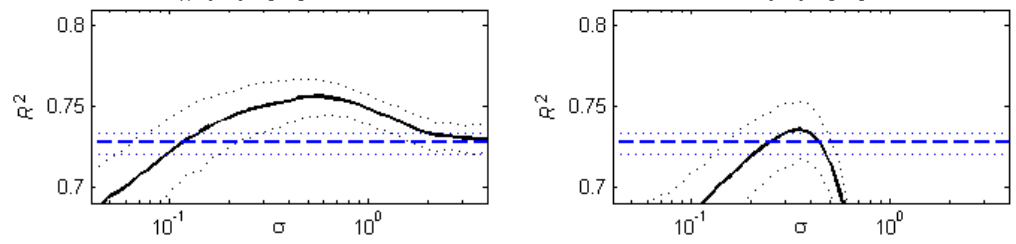

Fig. 6 continuation: Solid lines - mean $R^{2}$ calculated for the testing set (data not used for training the GRNN); dashed lines - mean $R^{2}$ calculated using LR; dotted lines $-5^{\text {th }}$ and $95^{\text {th }}$ percentiles of $R^{2}$. q-w: Results for the cascade GRNN without $\log E$ and $\log R$ as $\mathrm{GRNN}_{\mathrm{R}}$ inputs (Fig. 4c); x: Results for the GRNN replacing $\log R$ with station location and epicentral location as inputs.

The bootstrap analysis has confirmed that the best GMPE improvement is achieved when the epicenter location is used as an input (Figs. 6d, 61, and $6 \mathrm{~s})$. The improvement of the GMPE is also significant when only $\log E$ and $\log R$ are inputs for the GRNN, both for the separate GRNN (Fig. 6a) and for the cascade mode with the LR (Fig. 6i). The information about the station location improves the GMPE most when it is combined with $\log E$ and $\log R$ (Figs. 6b and 6j).

The test using only the station location in cascade with LR $\left(\operatorname{GRNN}_{\mathrm{R}}\left(x_{s}\right.\right.$, $\left.y_{s}\right)$ ) shows a small but statistically significant improvement of the GMPE 
(Fig. 6q). To assess this case, a GMPE model with relative local amplification factors (e.g. Lasocki 2013) was analysed and estimated using LR according to the formula:

$$
\log a_{i}=\alpha+\beta \log E+\gamma \log R+\sum_{j=1}^{J} w_{j} \delta_{i, j},
$$

where $i$ is the station number, $a_{i}$ are the $P H A$ values recorded by the $i^{\text {th }}$ station, $w_{i}$ is the estimated amplification factor at the $i^{\text {th }}$ station location, $\delta_{i, j}$ is Kronecker's delta, and $J=19$ is the number of stations. The other symbols are the same as in (9). $R^{2}=0.747$ was calculated for the tested data using a GMPE model with relative local amplification factors. The result is comparable with $\operatorname{GRNN}_{\mathrm{R}}\left(x_{s}, y_{s}\right)$.

In particular, the bootstrap test did not show a significant improvement for the model GRNN $\left(\log E, x_{\mathrm{e}}, y_{\mathrm{e}}, x_{s}, y_{s}\right)$, which did not consider the distance from the earthquake as an input (Fig. 6x). The results yielded by the holdout method were very good, whereas the bootstrap test did not show a significant improvement. This model either has low generalisation capability, or it is very sensitive to the reduction of the training set.

The application of the angular coefficients was based on the assumption that the GMPE is related to direction. An improvement of the GMPE in models with angular coefficients as the only inputs would suggest a constant trend for the whole zone. On the other hand, an improvement of the model in the case of angular coefficients in combination with the epicenter location would confirm that the earthquake mechanisms depend on location. The test results (Figs. 6o and 6v) show an improvement in the GMPE when $x_{\mathrm{e}}$ and $y_{\mathrm{e}}$ are added as inputs along with $\cos \varphi$ and $\sin \varphi$; however, the results are worse than for $x_{\mathrm{e}}$ and $y_{\mathrm{e}}$ alone. We can see, however, that $\mathrm{GRNN}_{\mathrm{R}}(\cos \varphi, \sin \varphi)$ requires a larger smoothness parameter (Fig. 6r) than $\operatorname{GRNN}_{\mathrm{R}}\left(x_{\mathrm{e}}, y_{\mathrm{e}}\right)$ (Fig. 6s). In addition, $R^{2}$ for larger values of $\sigma$ - optimum for $\operatorname{GRNN}_{\mathrm{R}}\left(x_{\mathrm{e}}, y_{\mathrm{e}}, \cos \varphi\right.$, $\sin \varphi$ ) (Fig. 6v) - is a little larger than $R^{2}$ in the model $\operatorname{GRNN}_{\mathrm{R}}\left(x_{\mathrm{e}}, y_{\mathrm{e}}\right)$. Thus, it cannot be ascertained whether the poorer results of the GMPE for the inputs $x_{\mathrm{e}}, y_{\mathrm{e}}, \cos \varphi$ and $\sin \varphi$ are a result of the lack of dependency, or of the unmatched inputs for one smoothness parameter.

Applying more inputs does not improve the results. The GRNN is most effective when only one additional input is incorporated to $\log R$ and $\log E$. In particular, combined information about the epicenters and directions did not improve the GMPE. It can be concluded that either focal directions in one place do not have any influence on the ground motion, or that this model of GRNN does not work properly with this combination of input variables. This could be overcome by using the input-dependent smoothing parameter, $\sigma$, which would further complicate the training of the GRNN, or by searching 
for a better metric $D$ to calculate a distance between samples of GRNN inputs.

\section{CONCLUSIONS}

Only one method of nonlinear estimation of GMPE by artificial neural networks was tested - the GRNN. Nonetheless, this approach proved that nonlinear regression modelled by artificial neural networks improves the GMPE.

The abilities of the GRNN were first analysed through learning, by employing a classic selection of the smoothness parameter using the holdout and bootstrap methods. The bootstrap tests showed that the results obtained from the classic selection using the holdout method are too optimistic, and the generalisation is in some cases not rewarding. The bootstrap method showed that some of the GRNN models significantly improved the GMPE, whereas others do not. This was not dependent on the value of the coefficient of determination. The bootstrap method has proved successful for verifying the GMPE model for some input sets, and for some of the GRNN models.

Various input variables for the GRNN were tested. The best results were obtained when one of the inputs was the location of the epicenter. The GMPE was also improved by applying to the GRNN inputs only the logarithms of distance and energy, which are commonly used in LR. A small but significant improvement in the GMPE was achieved when the station location was applied; the result was comparable to the GMPE calculated using the LR method with amplification factors. No significant improvement was achieved when the direction towards the earthquake source was taken as the input for the GRNN.

Out of all the models that were tested, the best results were obtained for a cascade connection of the GRNN with an LR of the logarithms of energy and distance, when these two values were also inputs of the GRNN. This model is superior to the separate GRNN, because the result is not as influenced by large values of the smoothness parameter. It is also superior to the cascade model without $\log E$ and $\log R$ as GRNN inputs, due to the nonlinear relationship between $\log$ PHA and the logarithms of energy and distance, which can be estimated by the two former models only.

The GRNN does not exhaust the possibilities of nonlinear methods of predicting ground motion. It was chosen to test whether using these kinds of methods is reasonable, because it is the fastest of the ANN methods, and the prediction is unique. Further studies are required in order to identify the most suitable method of estimating the GMPE in the LGCD. 


\section{Data and resources}

The data and ground motions used in this study were collected using a classified network of the KGHM Polska Miedź S.A, and cannot be released to the public.

Our calculations and figures were made using the Matlab program.

Acknowledgements. I wish to express gratitude to KGHM Polska Miedź S.A. - Oddział Zakład Hydrotechniczny for releasing the data for the study.

This work was partially supported within the statutory activity No. 3841/E-41/S/2015 of the Ministry of Science and Higher Education of Poland.

\section{References}

Abrahamson, N., and W. Silva (2008), Summary of the Abrahamson \& Silva NGA ground-motion relations, Earthq. Spectra 24, 1, 67-97, DOI: 10.1193/ 1.2924360 .

Akkar, S., and J.J. Bommer (2010), Empirical equations for the prediction of PGA, PGV, and spectra accelerations in Europe, the Mediterranean Region, and the Middle East, Seismol. Res. Lett. 81, 2, 195-206, DOI: 10.1785/ gssrl.81.2.195.

Arjun, C.R. (2013), Neural Network-Based Estimation of Strong Ground Motion Parameters, Lambert Academic Publishing, Saarbrücken.

Boore, D.M., and G.M. Atkinson (2008), Ground-motion prediction equations for the average horizontal component of PGA, PGV, and 5\%-damped PSA at spectral periods between $0.01 \mathrm{~s}$ and 10.0 s, Earthq. Spectra 24, 1, 99-138, DOI: $10.1193 / 1.2830434$.

Campbell, K.W., and Y. Bozorgnia (2008), NGA ground motion model for the geometric mean horizontal component of PGA, PGV, PGD and 5\% damped linear elastic response spectra for periods ranging from 0.01 to $10 \mathrm{~s}$, Earthq. Spectra 24, 1, 139-171, DOI: 10.1193/1.2857546.

Cornell, C.A. (1968), Engineering seismic risk analysis, Bull. Seismol. Soc. Am. 58, 5, 1583-1606.

Derras, B., and A. Bekkouche (2011), Use of the Artificial Neural Network for Peak Ground Acceleration estimation, Lebanese Sci. J. 12, 2, 101-115.

Derras, B., P.-Y. Bard, F. Cotton, and A. Bekkouche (2012), Adapting the neural network approach to PGA prediction: An example based on the KiK-net data, Bull. Seismol. Soc. Am. 102, 4, 1446-1461, DOI: 10.1785/ 0120110088. 
Douglas, J. (2011), Ground-motion prediction equations 1964-2010, BRGM/RP59356-FR, 444 pp.

Efron, B. (1979), Bootstrap methods: another look at the jackknife, Ann. Stat. 7, 1, 1-26, DOI: $10.1214 / \mathrm{aos} / 1176344552$.

García, S.R., M.P. Romo, and N. Sarmiento (2003), Modeling ground motion in Mexico City using artificial neural networks, Geofís. Int. 42, 2, 173-183.

Golik, A., and M.J. Mendecki (2012), Ground-motion prediction equations for induced seismicity in the main anticline and main syncline, Upper Silesian Coal Basin, Poland, Acta Geophys. 60, 2, 410-425, DOI: 10.2478/s11600011-0070-9.

Güllü, H., and E. Erçelebi (2007), A neural network approach for attenuation relationships: An application using strong ground motion data from Turkey, Eng. Geol. 93, 3, 65-81, DOI: 10.1016/j.enggeo.2007.05.004.

Günaydin, K., and A. Günaydin (2008), Peak ground acceleration prediction by artificial neural networks for northwestern Turkey, Math. Problems Eng. 2008, article ID 919420, 1-20, DOI: 10.1155/2008/919420.

Hanna, A.M., D. Ural, and G. Saygili (2007), Neural network model for liquefaction potential in soil deposits using Turkey and Taiwan earthquake data, Soil Dyn. Earthq. Eng. 27, 6, 521-540, DOI: 10.1016/j.soildyn.2006.11.001.

Hong, H., T. Liu, and C.-S. Lee (2012), Observations on the application of artificial neural network to predicting ground motion measures, Earthq. Sci. 25, 2, 161-175, DOI: 10.1007/s11589-012-0843-5.

Joyner, W.B., and D.M. Boore (1988). Measurement, characterization, and prediction of strong ground motion. In: Proc. Earthquake Engineering \& Soil Dynamics II. Geotechnical Division, ASCE Special Publication 20, 43-102.

Joyner, W.B., and D.M. Boore (1993), Methods for regression analysis of strongmotion data, Bull. Seismol. Soc. Am. 83, 2, 469-487.

Lasocki, S. (2013), Site specific prediction equations for peak acceleration of ground motion due to earthquakes induced by underground mining in LegnicaGłogów Copper District in Poland, Acta Geophys. 61, 5, 1130-1155, DOI: 10.2478/s11600-013-0139-8.

Moody, J. (1994), Prediction risk and architecture selection for neural networks. In: J. Cherkassy, J.H. Friedman, and H. Wechsler. (eds.), From Statistics to Neural Networks: Theory and Pattern Recognition Applications, NATO ASI Series, Springer, Berlin, 147-165, DOI: 10.1007/978-3-642-79119-2.

Parzen, E. (1962), On estimation of a probability density function and mode, Ann. Math. Statist. 33, 3, 1065-1076, DOI: 10.1214/aoms/1177704472.

Pozos-Estrada, A., R. Gómez, and H.P. Hong (2014). Use of neural network to predict the peak ground accelerations and pseudo spectral accelerations for Mexican Inslab and Interplate Earthquakes, Geofís. Int. 53, 1, 39-57, DOI: 10.1016/S0016-7169(14)71489-8. 
Specht, D.F. (1991), A general regression neural network, IEEE Trans. Neural Network 2, 6, 568-576, DOI: 10.1109/72.97934.

Trifunac, M.D., and A.G. Brady (1976), Correlations of peak acceleration, velocity and displacement with earthquake magnitude, distance and site conditions, Earthq. Eng. Struct. Dyn. 4, 5, 455-471, DOI: 10.1002/eqe.4290040504.

Wasserman, P.D. (1993). Advanced Methods in Neural Computing, John Wiley \& Sons, Inc. New York.

Yaghmaei-Sabegh, S. (2012). A new method for ranking and weighting of earthquake ground-motion prediction models, Soil Dyn. Earthq. Eng. 39, 78-87, DOI: $10.1016 / j$.soildyn.2012.03.006.

Yaghmaei-Sabegh, S., and H.-H. Tsang (2011), A new site classification approach based on neural networks, Soil Dyn. Earthq. Eng. 31, 7, 974-981, DOI: 10.1016/j.soildyn.2011.03.004.

Yaghmaei-Sabegh, S., and H.-H. Tsang (2014), Site class mapping based on earthquake ground motion data recorded by regional seismographic network, Nat. Hazards 73, 3, 2067-2087, DOI: 10.1007/s11069-014-1177-5.

Received 3 December 2015

Received in revised from 18 April 2016 Accepted 26 July 2016 